\title{
Two Treatment Approaches for Missing Maxillary Lateral Incisors: A Case
}

\author{
Abdulgani Mai*, Abdulgani Azzaldeen, Watted Nezar, Abu-Hussein Muhamad
}

\begin{abstract}
Missing maxillary lateral incisors create an esthetic problem with specific orthodontic and prosthetic considerations. The aim of the present study is to evaluate the clinical success of the transmucosal flapless implant placement and immediate loading of the implants to restore the agenic lateral incisors after completing the orthodontic treatment and during the retention period.
\end{abstract}

Keywords: Dental implant, lateral incisor, case report.

\section{Introduction}

Dental agenesis is defined as congenital absence of one or more teeth in primary or permanent dentition. It is also known as hypodontia and is one of the most frequently encountered of all oral variation that affects a large population. [1]Epidemiological studies reveal make that one of the most common congenitally missing tooth is lateral incisor in maxilla causing esthetic and functional impairments in the affected individual. [1,2] It might be associated with Non-syndromic systemic problems, syndromic conditions or other oral anmolaies. Management of missing lateral incisors is a challenging procedure that involves a multi-disciplinary approach for rehabilitation of impaired esthetics and function.[1,2,3]

The most common treatment approaches advocated by the clinicians include regaining of the space of missing tooth followed by prosthetic replacement, auto transplantation of developing premolar and space closure with substitution of canine.

Missing maxillary lateral incisors create an esthetic problem with specific orthodontic and prosthetic considerations. Dental implants are commonly used to replace congenitally missing lateral incisors in young orthodontic patients. [1,2,3,4]However, an interdisciplinary approach should be observed during the diagnosis,prognosis, and treatment plan to provide a result with good predictability, reaching the esthetic and functional expectations of the patient .[1]

In population studies, the most commonly missing tooth, excluding third molars, is the mandibular second premolar followed by the maxillary lateral incisor . [5]There is a range of reported values for prevalence of hypodontia due to heterogeneous study designs: sample size, gender distribution of sample, records used to determine missing teeth and geographic differences reflecting ethnically distinct populations. A meta-analysis revealed that dental agenesis is 1.37 times more common in females than males . [5]The prevalence of missing maxillary lateral incisors ranges from $0.95 \%$ in an American Caucasian sample to $2 \%$ in an Icelandic sample . A meta-analysis of ten studies, totaling 48274 subjects, found the prevalence of missing maxillary lateral incisors to be $1.6 \%$. Orthodontists can expect a greater percentage of their patients to present with the chief complaint of missing maxillary lateral incisors. In one sample of orthodontic patients, missing teeth more commonly occurred in the maxilla with the maxillary lateral incisors cited to be the most commonly missing teeth.$[5,6]$ This is likely related to the heightened awareness of the esthetic problem of a missing tooth in the maxillary anterior region.[6,7]

Dental agenesis has been attributed to genetic and environmental factors. The genetic background is involved in the majority of cases. In fact, the expression of more than 200 genes is responsible for tooth development, so a mutation in any of these can arrest this process. Familiar dental agenesis may be inherited in an autosomal (dominant or recessive) or X-linked (dominant or recessive) pattern. Moreover, both the genotype and phenotype of dental agenesis, in general, present heterogeneity.39 To date, only the cases of severe dental agenesis with dominant inheritance and syndromic dental agenesis with recessive inheritance are well known as the result of specific genetic defects.39 The most common types of dental agenesis maxillary lateral incisors and premolars are merely identified.[3.8,9]

Brook [10] suggests that most cases of hypodontia have a polygenetic inheritance pattern and that the risk of relatives having hypodontia will depend on a combination of numerous genetic and environmental factors. As well as the familial nature of hypodontia, it often presents as an isolated diagnosis with no detectable family history, which suggests it can occur as a result of a spontaneous genetic mutation. Hypodontia has also been associated with cleft lip and palate usually localized to the maxillary lateral incisor in the line of the alveolar cleft. [11.12] 

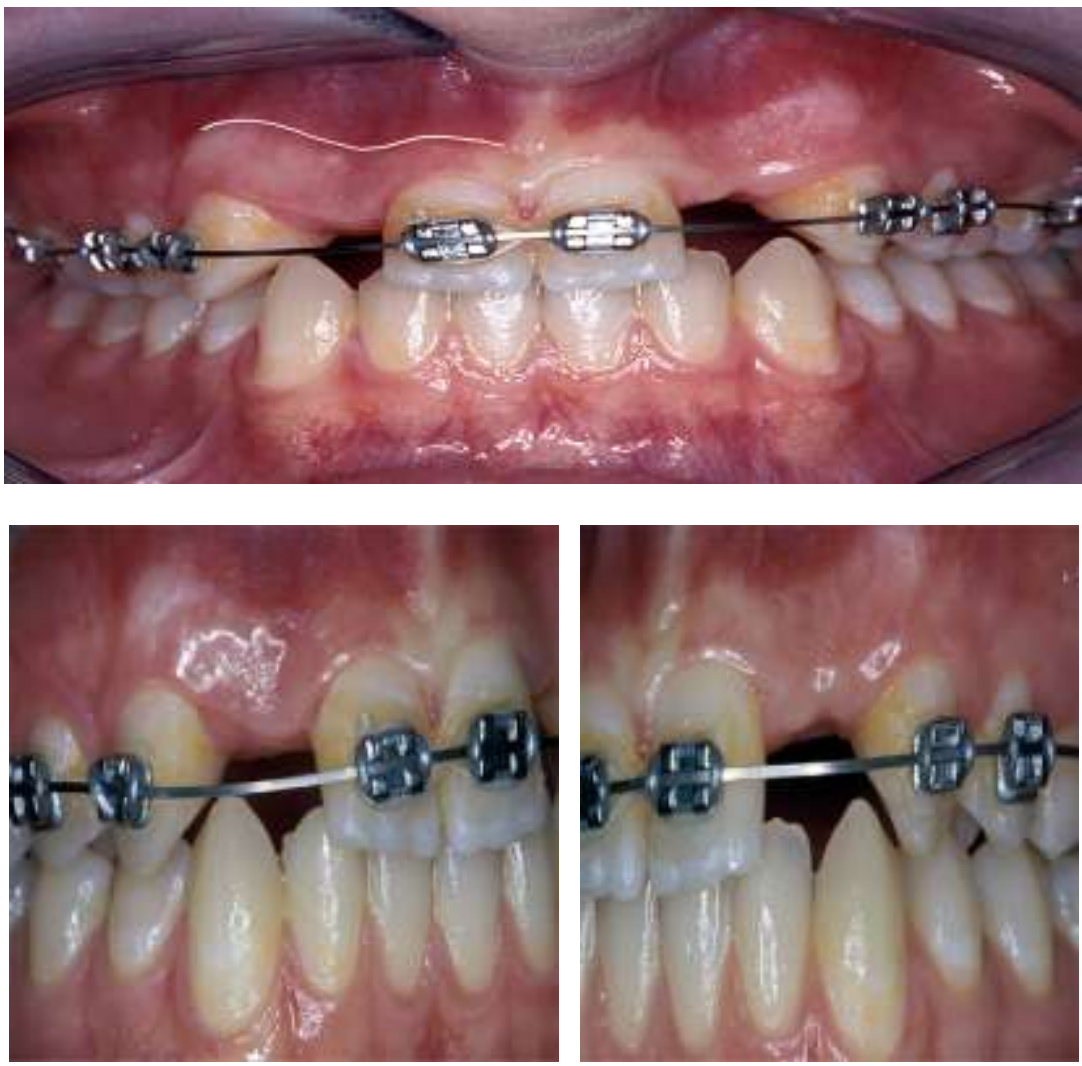

Fig.1a-c ; Pretreatment buccal views showed (A) root convergence, (B) a difference in clinical appearance of the left lateral, and (C) significant crestal width of keratinized tissue.

Hypodontia creates significant challenges to the clinicians in both diagnosis and management. Comprehensive management often requires a multidisciplinary approach.[12.13,14[

There are different treatment alternatives for patients with a missing lateral incisor because of congenital reasons . Esthetic and functional problems can arise when an orthodontic space closure is realized and the canine is moved into the missing lateral incisor's space.[12.13.14.15]

The aim of the present study is to evaluate the clinical success of the transmucosal flapless implant placement and immediate loading of the implants to restore the agenic lateral incisors after completing the orthodontic treatment and during the retention period.

\section{Case Report}

The initial clinical exam revealed diastema, congenitally missing maxillary lateral incisors with the canines located in the lateral incisor positions, and the primary maxillary canines still located in their original positions.These aspects created not only esthetics deficiencies but also maloclussion. Therefore, a multidisciplinary treatment was suggested to restore both esthetics and function. Fig.1a-c

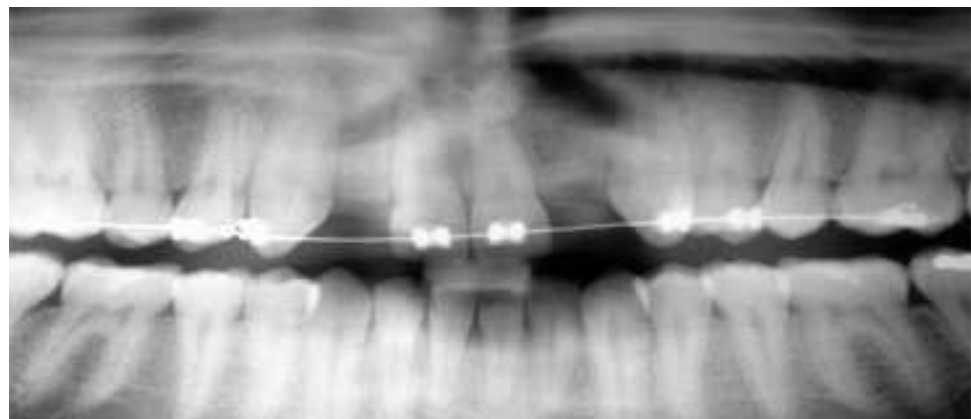

Fig. 2a; After orthodontic therapy, the radiograph did not provide enough diagnostic information to determine if implants could be placed 


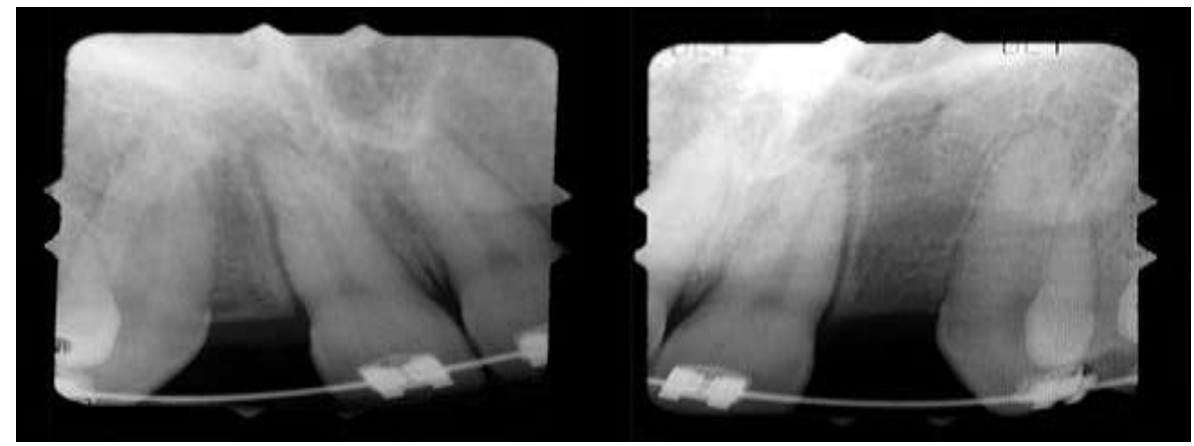

Fig. 2b; Pretreatment radiographs revealed convergent roots for the right canine and right central

The patient is a 17-year-old Arab Israeli girl. Presenting complaints are localized spacing and tilting of upper canines. She has no previous orthodontic treatment history. The pretreatment extra oral photographs show a concave facial profile (Fig. 1a-c). The intraoral examination showed a Class I molar relationship of 46 and Class II molar relationship of 36. All permanent teeth were erupted except maxillary lateral incisors (12 and 22). Both maxillary canines (13 and 23) had tilted mesially creating spaces distally . 11 was in single tooth crossbite and a reduced overbite of the 21 was identified. On radiographic examination, 12 and 22 were absent and the roots of maxillary canines were present distally whereas only crowns were tipped mesially towards 11 and 21 respectively (Fig.2). The inclination of the maxillary incisors was normal, but the mandibular incisors showed a lingual inclination (Fig. 2a-b). Soft tissue analysis showed protrusive lower lip.
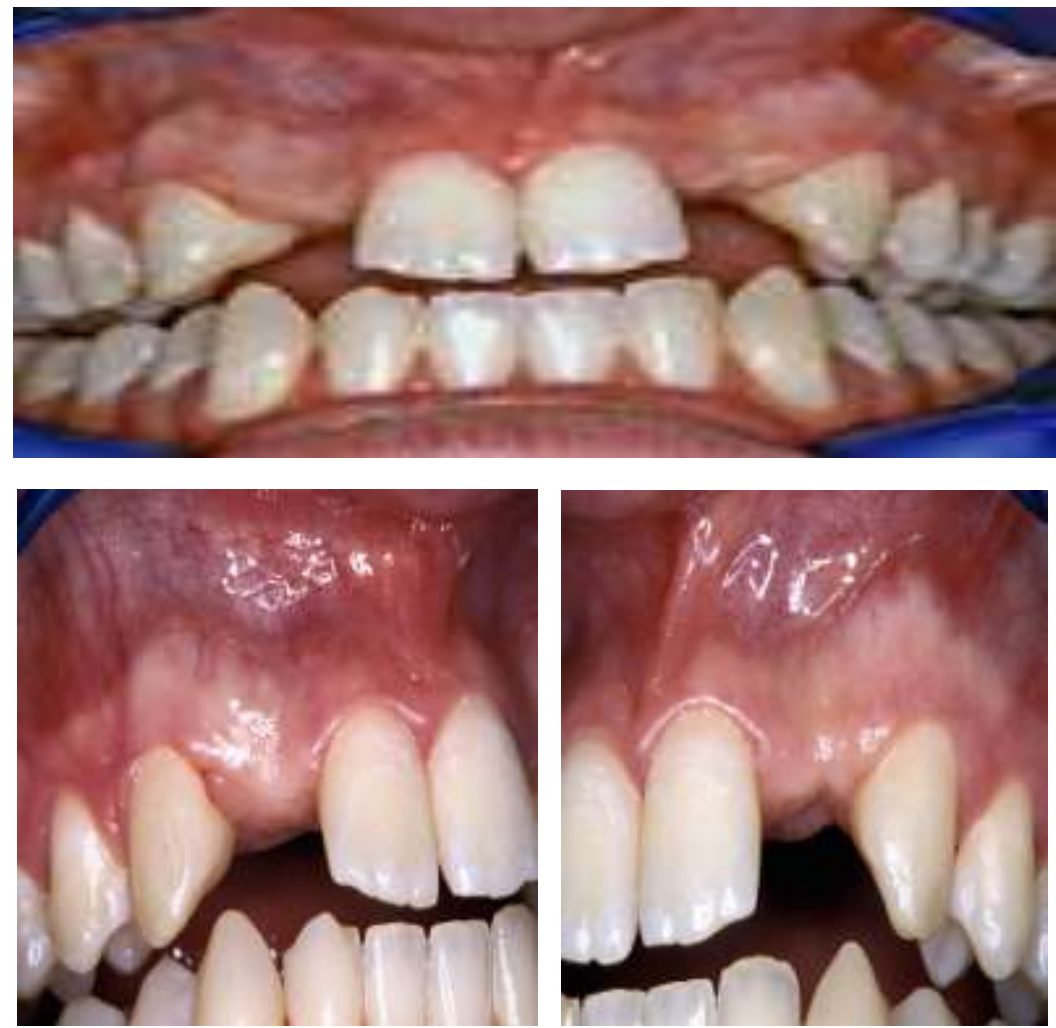

Fig 3a-c; Before implant surgery, the orthodontic brackets were removed, revealing (A) the position of the centrals, and $(\mathrm{B}, \mathrm{C})$ the anatomical variations of the crestal tissue and lack of interdental papilla

\section{Treatment Progress}

\section{Orthodontic Part}

Conventional fixed appliance ( $0.022 \times 0.028 \mathrm{in})$ slot were first placed in upper arch and leveling alignment was started by $0.012 \mathrm{NiTi}$. Then $0.016 \mathrm{NiTi}, 017 \mathrm{X} 024 \mathrm{NiTi}$ and $018 \mathrm{SS}$ were installed sequentially. $0.016 \mathrm{NiTi}, 0.018 \mathrm{NiTi}, 0.017 \mathrm{X} 0.025 \mathrm{NiTi}$, and $018 \mathrm{SS}$ were installed in lower arch chronologically. Crossbite was corrected using fixed orthodontic treatment. The mesially tilted maxillary canines were aligned distally to create spaces for maxillary lateral incisors. The canines and central incisors were aligned in the dental arch. (Fig. 1a-c,Fig .3a-c) 


\section{Surgical Part}

After 15 months of orthodontic treatment, exact locations for surgical implant placement were determined by CBCT 3-D positioning system. $11,5 \mathrm{~mm}$ implant for 11 and $13 \mathrm{~mm}$ implant for 21 , were placed. The temporary crowns were inserted while waiting for the healing process. Fig.4.5

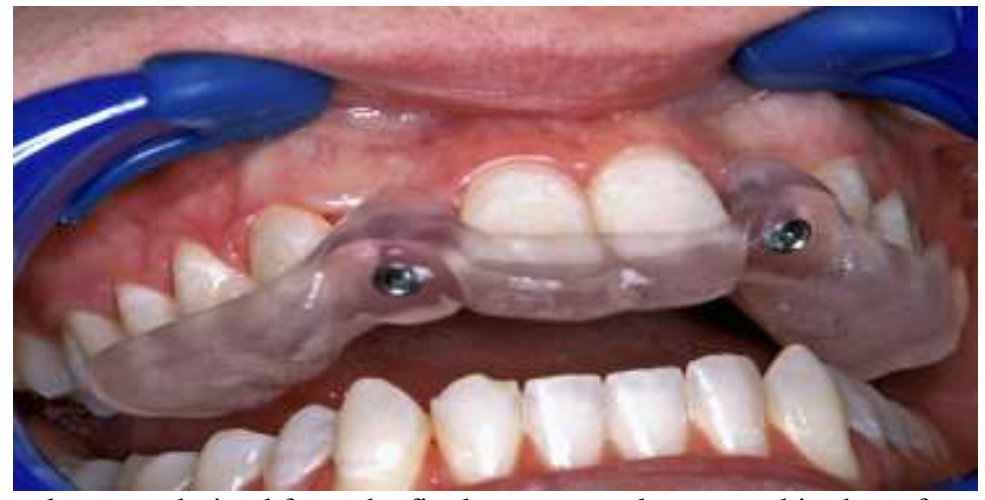

Fig.4; A surgical template was derived from the final treatment plan created in the software, which facilitated (A) the drilling sequence and (B) precise location of placement

\section{Prosthodontic Part}

Temporary prostheses were inserted to maintain and prevent vertical space loss of final crown. Final prostheses were inserted after six months of surgery for the optimal osseointegration and Vacuum form clear retainer were initially fabricated. Then passive Hawley's retainers were given for maintenance of final finishing. Fig. 6,7

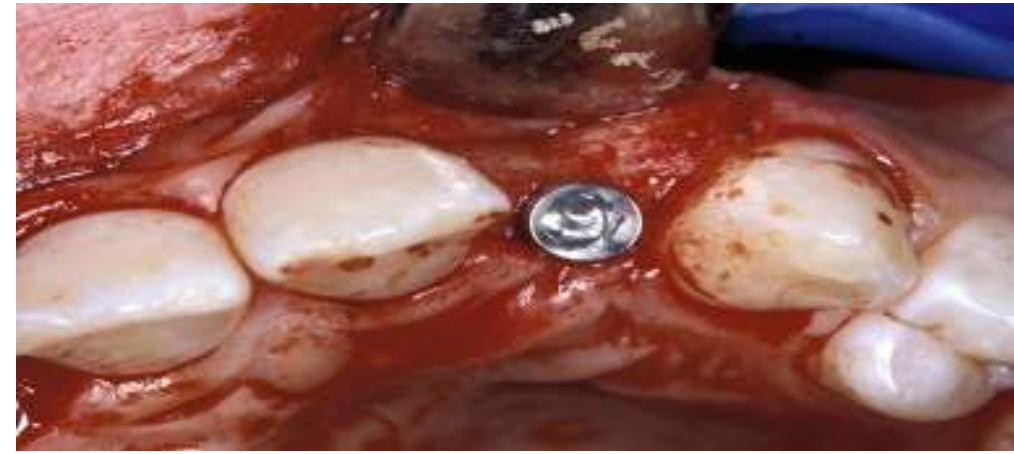

Fig.5; After the osteotomies were completed, the implants were placed.

\section{Treatment Results}

Post-treatment visit confirmed that patient's complaints had been addressed. Teeth were esthetically pleasing. Upper and lower arches were well aligned (Fig.3). Appropriate distallization of canines created spaces required for implantation of missing maxillary lateral incisors. Posterior occlusion had good overall intercuspation and was well settled in a Class I molar relationship. Crossbite of mandibular central incisor had been corrected after which normal overjet and overbite were achieved. Upper and lower dental midlines were coincident with facial midline. Fig. 7,8

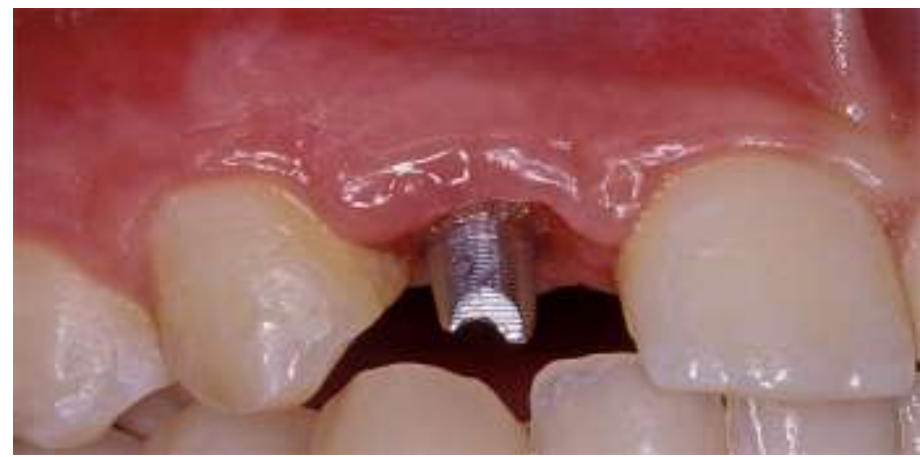

Fig.6; The patient- abutments were placed 


\section{Discussion}

Agenesis of teeth can be due to genetic or environmental factors. In non syndromic agenesis, gene mutations are said to be the cause. The mutations in genes responsible for tooth development are marked as PAX9, MSX1, and AXIN2.11 Among these, MSX1 is usually related to congenitally missing third molars, second premolars, maxillary first premolar and incisors. [3,8]

A variety of esthetic problems are associated with maxillary lateral incisor agenesis. This includes median diastema, spacing between permanent incisor and canine, mesial migration of canines, midline shift in case of unilateral missing tooth.[1,2] Over-retention of maxillary deciduous lateral incisor and canine, ectopically erupted canines, absence of canine eminence, asymmetric loss of primary teeth, dental asymmetries are significant apparent factors that are useful for diagnosing the entity.

Confirmed diagnosis of a missing tooth requires radiograph like periapical view or an OPG. Among them, tomography is the most reliable method for diagnosing congenitally missing teeth.[1.2,6.9]

Management of missing maxillary lateral incisors is challenging process for re-establishment of esthetics and function of the affected individuals. The most appropriate approaches as reported in the literature namingly are i) Regaining of space by orthodontic therapy ii) Autotransplantaion of premolars iii) Orthodontic space closure. $[9,12]$

1) Regaining of space by orthodontic therapy: this approach is aimed to provide adequate space for replacement of missing tooth. Space opening can be achieved by closing the midline diastema and retracting the ectopically erupted canines. The amount of space required can be determined by application of golden proportions, measuring the Bolton discrepancy and the missing lateral incisor is then replaced to the best of its esthetic and functional demand using a variety of alternatives as per patients choice and socioeconomic status. These include removable denture, fixed cantilever bridge, fiber reinforced composite fixed partial dentures, resin bonded fixtures, or by implant supported restoration.

2) Autotransplantaion of premolars: Autotransplantaion of premolars at maxillary lateral incisor position is suggested when $2 / 3$ rd root of premolar roots have developed so that autotransplanted teeth can achieve functional adjustment. The success rates of autotransplantaion range from $79-90 \%$ as reported in the literature. After three months of successful autotransplantaion, the crown of premolar is modified to bear a resemblance with the maxillary lateral incisor both esthetically and functionally.

3) Orthodontic space closure: It is best indicated for patients presenting a class II malocclusion with noncrowded lower arch, class I malocclusion with sever crowded upper lower arches where extractions are required and in cases where upper anterior teeth are proclined. This approach involves orthodontics space closure by moving the canines to missing lateral incisor place and making contact with central incisors. After orthodontic space closure, the crowns of canine and premolars are modified to take esthetic and functional semblance of lateral incisor. This approach also has an advantage that normal gingival and alveolar bone architecture can be maintained by mesial movement of teeth into the available space, prosthetic replacement can be avoided and possibility of third molar impaction is also decreased

However, following this method would result in anterior group functioning during lateral excursions instead of canine guided occlusion. This occlusal scheme is also acceptable and considered stable by several school of thoughts .[1,2,4,6]

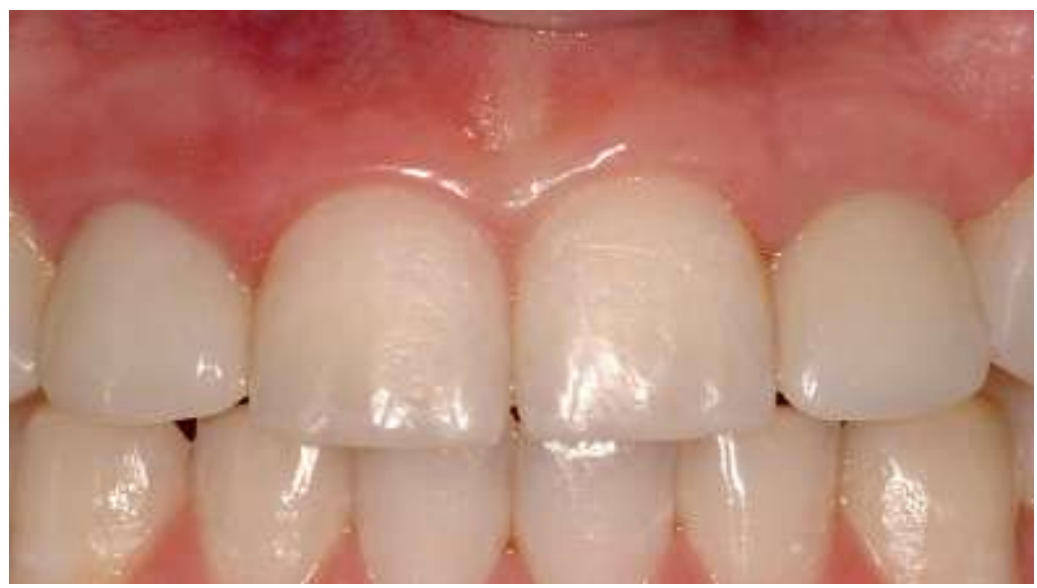

Fig.7; The final restorations are indistinguishable from the surrounding dentition, demonstrating good emergence and re-creation of the interdental papillae 
Each one has its own indications, advantages and disadvantages. Osseointegrated implants may be used to restore single unit spaces including the congenitally missing maxillary lateral incisor. However, implants are not indicated in patients for whom a potential for further growth exists since an implant, which does not have periodontal ligament, cannot erupt and keep pace with dentoalveolar development . Single tooth implants are likely to be of most use in the adult patients.[1,2,9] Patient selection should be considered carefully keeping in mind oral hygiene socio-economic background and ability of patient to follow maintenance instructions. The importance of early diagnosis of missing teeth should not be forgotten, so that a multidisciplinary approach can be established at an early stage. Preparatory orthodontic treatment may be needed to align teeth, create adequate space in addition to establish an optimal axial inclination of the teeth adjacent to the proposed implant site. With proper patient selection and diagnosis, understanding of occlusion, effective communication among operators and attention to detail, the single tooth restoration can be a predictable esthetic and longlasting restorations .In this case, alveolar bone was available in maxillary lateral incisor areas in the mesiodistal and coronoapical dimension; however, there was deficiency in orofacial dimension.[1,2,4, 6.7,9]

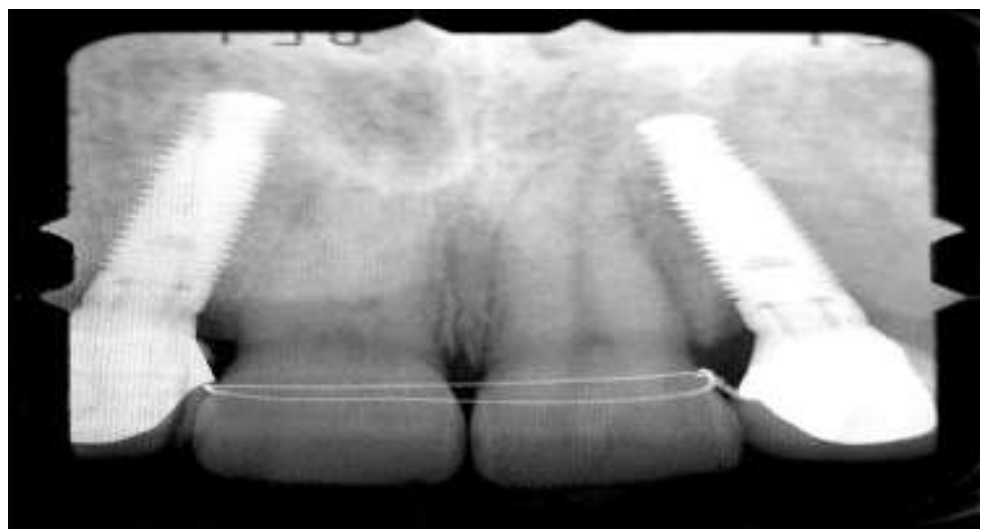

Fig.8; The final radiograph confirmed the fit of the abutment-implant-crown interfaces

Implants are commonly used to replace congenitally missing lateral incisors in adolescent orthodontic patients. However, these restorations are often challenging for the orthodontist, surgeon, and restorative dentist. In some patients the space across the alveolar crest is too narrow to permit the surgeon to place the implant. Occasionally the root apices of the adjacent central incisor and canine are in close proximity. In other cases the ridge thickness could be inadequate and require augmentation. When the orthodontist opens the space, the papilla heights are adversely affected. Some adolescent patients have altered passive eruption after orthodontic treatment, which affects the level of the gingivalmargins.[10,11,12,13,14]

For patients with congenitally missing lateral incisors, in addition to over-retained primary teeth, permanent canines may erupt or drift mesially into the edentulous space. If the space is to be opened orthodontically for ideal prosthesis, the canines $\mathrm{vb}$ will need to be moved distally, which may result in development of the alveolar ridge in the canine region [ $15,16,17,18]]$. In cases where the occlusion and esthetics of the canine in the lateral position are acceptable, closure of the lateral space by the mesially positioned canine may be the simplest alternative treatment option.However, in all the above congenital missing cases we considered space opening followed by fixed prosthesis would be more acceptable on aesthetic point of view [19,20,21]. Esthetics as well as occlusion must be considered in the final orthodontic positioning of the teeth adjacent to the edentulous space. To satisfy the "golden proportion" principle of esthetics, the space for the maxillary lateral incisor should be approximately two-thirds of the width of the central incisor.[9] However, if the patient is missing only one maxillary lateral incisor, the space required to achieve symmetrical esthetics and occlusion is primarily dictated by the width of the contralateral incisor [22]. The optimal time for placement of fixed prosthesis is after the growth of the maxilla, mandible and alveolus is complete. If fixed prosthesis/implant are placed before growth is complete, the surrounding alveolar bone may continue to develop vertically and adjacent teeth may continue to erupt. Thus a discrepancy between the gingival margins of the prosthesis and the natural teeth is created and the prosthesis appears to became submerged. This creates a functional as well as an esthetic problem [23].

In this case, alveolar bone was available in maxillary lateral incisor areas in the mesiodistal and coronoapical dimen-sion; however, there was deficiency in orofacial dimension. The patient was refused to have bone augmentation procedures using either autogenic or synthetic bone grafts because of financial and patient 
related factors . Therefore, implants with $3 \mathrm{~mm}$ diameter were used to compensate for horizontal alveolar bone deficiency. However, to avoid labial fenestration, the implants had to be placed off axis in labial direction. The relationship of the position between the implant and the proposed restoration should be based on the position of the implant shoulder, since it will influence the final hard and soft tissue response . The malposition of the implant shoulder in the coronoapical direction causes soft tissue recession. In this case, location of the implant shoulders was in coronoapical and mesiodistal dimension in comfort zone. However, in the orofacial dimension the implant shoulders were in danger zone. The angulation of implants in labial direction was compensated using angled abutments that were prepared for better emergence profile of the ceramic crowns. Many authors have also concluded that angled abutments may be considered a suitable restorative option when implants are not placed in ideal axial positions. Nevertheless, forces applied off axis may be expected to overload the bone surrounding single-tooth implants, as shown by Papavasiliou et al using finite element analysis. Hence, the segmental osteotomy may provide an alternative treatment to reposition the severely malposed implants.[25] Finally, questions commonly arise regarding the appropriate age for implant placement in these young patients. If not addressed, these issues could compromise the aesthetics of the implant restoration . From an aesthetic and functional point of view such patients need a combination of orthodontic, prosthetic and restorative care.

In this case, the use of a simple vacuum formed surgical guide template was found to be very cost effective and accurate for the implant placement.[1,9]

The decision to open or to close absent maxillary lateral incisor spaces remains a challenging task for most orthodontists. The clinician must balance the advantage of avoiding long-term restorations with the esthetic limitations imposed by using canines as lateral incisor substitutes. All efforts from the professionals involved should focus on minimizing the treatment margin of error by developing good coordination among the multidisciplinary team members, capitalizing on their experience, and consequently achieving an individualized treatment plan that efficiently meets the patient's expectations.

\section{Conclusions}

Maxillary lateral incisor is among one of the most common congenitally missing teeth that occurs either due to genetic or environmental disturbances. Management of missing lateral incisors is a challenging and complex process that involves a multidisciplinary approach in order to restore the esthetics and function. Emphasis on early diagnosis with a team consultation followed by a timely planned and well executed inter-disciplinary treatment plan is the ultimate solution for such complex cases.

\section{References}

[1]. Abu-Hussein, M., Watted, N., Abdulgani, A., and Bajali, M. Treatment of Patients With Congenitally Missing Lateral Incisors: Is an Interdisciplinary Task, RRJDS2014, 2(4),53-68

[2]. Muhamad AH, Azzaldeen A, Nezar W, Mohammed Z. Esthetic Evaluation of Implants Placed after Orthodontic Treatment in Patients with Congenitally Missing Lateral Incisors. J Adv Med Dent Scie Res 2015;3(3):110-118.

[3]. Abu-Hussein M., Watted N., Yehia M., Proff P., Iraqi F.; Clinical Genetic Basis of Tooth Agenesis, Journal of Dental and Medical Sciences2015,14(12),68-77

[4]. Abu-Hussein M., Abdulgani A., Watted N .Zahalka M.; Congenitally Missing Lateral Incisor with Orthodontics, Bone Grafting and Single-Tooth Implant: A Case Report. Journal of Dental and Medical Sciences2015, 14(4),124-130

[5]. Polder BJ, Van't Hof MA, Van der Linden FP, Kuijpers-Jagtman AM. A meta-analysis of the prevalence of dental agenesis of permanent teeth. Community Dent Oral Epidemiol. 2004;32:217-26.

[6]. Abu-Hussein M., Watted N., Watted A., Abu-Hussein Y, Yehia M .Awadi O. , Abdulgani A .; Prevalence of Tooth Agenesis in Orthodontic Patients at Arab Population in Israel, International Journal of Public Health Research ,2015; 3(3): 77-82.

[7]. Muhamad Abu-Hussein, Nezar Watted, Abdulgani Azzaldeen, Mohammad Yehia, Obaida Awadi, Yosef Abu-Hussein. Prevalence of Missing Lateral Incisor Agenesis in an Orthodontic Arabs Population in Israel (Arab48). International Journal of Public Health Research. Vol. 3, No. 3, 2015, pp. 101-107.

[8]. Abu-Hussein M., Watted N., Abdulgani N. , Alterman M.; Non-Syndromic Oligodontia: A Rare Case Report, jmscr2015,3(5), . 5649-5655

[9]. Abu-Hussein M., Watted N., Abdulgani A., BorbélyB.; Modern Treatment for Congenitally Missing Teeth : A MultidisciplinaryApproach; INTERNATIONAL JOURNAL OF MAXILLOFACIAL RESEARCH,2015,1(2);179-190

[10]. Brook AH. A unifying aetiological explanation for anomalies of human tooth number and size. Arch Oral Biol 1984, 29(5):373-378

[11]. Dhanrajani PJ. Hypodontia: Etiology, clinical features, and management. Quintessence Int 2002, 33:294-302

[12]. Abu-Hussein M, Watted N, ; Congenitally Missing Upper Laterals. Clinical Considerations: Orthodontic Space Closure,Int Res Pub Med Sci, 2015;1(3):82-89

[13]. Abu-Hussein M., Watted N., Abdulgani A., Kontoes N.; Prosthodontic-Orthodontic Treatment Plan with Two-UnitCantilevered Resin-Bonded Fixed Partial Denture, IOSR-JDMS 2015,14(12) , 131-136

[14]. Abdulgani A.,. Kontoes N., Chlorokostas G.,Abu-Hussein M .; Interdisciplinary Management Of Maxillary Lateral Incisors Agenesis With Mini Implant Prostheses: A Case Report; IOSR-JDMS 2015,14 (12), 36-42 
[15]. Abusalih A. , Ismail H, Abdulgani A., Chlorokostas G ., Abu-Hussein M .; Interdisciplinary Management of Congenitally Agenesis Maxillary Lateral Incisors: Orthodontic/Prosthodontic Perspectives, Journal of Dental and Medical Sciences2016, Vol 15, $1,90-99$

[16]. Kinzer GA, Kokich VO. Managing congenitally missing lateral incisors. Part I: canine substitution. J Esthet Restor Dent. $2005 ; 17: 5-10$

[17]. Kokich OV, GA Kinzer and J Janakievski. Congenitally missing maxillary lateral incisors: Restorative replacement. American J Orthodont Dentofacial Orthop. 2011;139(4):435-445

[18]. Gumus, H.O., N. Hersek, I. Tuluoglu and F. Tasar, . Management of Congenitally Missing Lateral Incisors with Orthodontics and Single-Tooth Implants: Two case Reports. Dental Res J. 2008, 5(1): 37-40.

[19]. Olsen TM, Kokich VG Sr. Postorthodontic root approximation after opening space for maxillary lateral incisor implants. Am J Orthod Dentofacial Orthop. 2010;137:158-9.

[20]. Sabri R. Management of missing lateral incisors. J Am Dent Assoc. 1999, 130:80-84

[21]. Richardson G, Russell KA. Congenitally missing maxillary lateral incisors and orthodontic treatment considerations for the singletooth implant. J Can Dent Assoc. 2001;67(1):25-8.

[22]. Winkler S, Boberick KG, Braid S, Wood R, Cari MJ. Implant replacement of congenitally missing lateral incisors: a case report. J Oral Implantol. 2008;34(2):115-18.

[23]. Tuna SH, Keyf F, Pekkan G. The single-tooth implant treatment of congenitally missing maxillary lateral incisors using angled abutments: a clinical report. Dent Res J (Isfahan) 2009;6(2):93-98.

[24]. Wheeler, S.L., Implant complications in the esthetic zone. J Oral Maxillofac. Surg; Suppl 1,2007; 65: 93-102

[25]. Papavasiliou G, Kamposiora P, Bayne SC, Felton DA. Three-dimensional finite element analysis of stress-distribution around single tooth implants as a function of bony support, prosthesis type, and loading during function. J Prosthet Dent. 1996;76(6):633-40 